\title{
Screening models using multiple markers for early detection of late-onset preeclampsia in low-risk pregnancy
}

Hee Jin Park', Soo Hyun Kim', Yong Wook Jung ${ }^{1}$, Sung Shin Shim', Ji Yeon Kim', Yeon Kyung Cho', Antonio Farina ${ }^{2}$, Margherita Zanello ${ }^{2}$, Kyoung Jin Lee ${ }^{1+}$ and Dong Hyun Cha $a^{1,3^{*+}}$

\begin{abstract}
Background: Our primary objective was to establish a cutoff value for the soluble fms-like tyrosine kinase 1(sFlt-1)/ placental growth factor (PIGF) ratio measured using the Elecsys assay to predict late-onset preeclampsia in low-risk pregnancies. Our secondary objective was to evaluate the ability of combination models using Elecsys data, second trimester uterine artery (UtA) Doppler ultrasonography measurements, and the serum fetoplacental protein levels used for Down's syndrome screening, to predict preeclampsia.

Methods: This prospective cohort study included 262 pregnant women with a low risk of preeclampsia. Plasma levels of pregnancy-associated plasma protein-A (PAPP-A) and serum levels of alpha-fetoprotein, unconjugated estriol, human chorionic gonadotropin, and inhibin-A were measured, and sFlt-1/PIGF ratios were calculated. All women underwent UtA Doppler ultrasonography at 20 to 24 weeks of gestation.
\end{abstract}

Results: Eight of the 262 women (3.0\%) developed late-onset preeclampsia. Receiver operating characteristic curve analysis showed that the third trimester sFIt-1/PIGF ratio yielded the best detection rate (DR) for preeclampsia at a fixed false-positive rate (FPR) of 10\%, followed by the second trimester sFlt-1/PIGF ratio, sFlt-1 level, and PIGF level. Binary logistic regression analysis was used to determine the five best combination models for early detection of late-onset preeclampsia. The combination of the PAPP-A level and the second trimester sFlt-1/PIGF ratio yielded a DR of $87.5 \%$ at a fixed FPR of 5\%, the combination of second and third trimester sFIt-1/PIGF ratios yielded a DR of $87.5 \%$ at a fixed FPR of $10 \%$, the combination of body mass index and the second trimester sFlt-1 level yielded a DR of $87.5 \%$ at a fixed FPR of 10\%, the combination of the PAPP-A and inhibin-A levels yielded a DR of $50 \%$ at a fixed FPR of 10\%, and the combination of the PAPP-A level and the third trimester sFlt-1/PIGF ratio yielded a DR of $62.5 \%$ at a fixed FPR of $10 \%$.

Conclusions: The combination of the PAPP-A level and the second trimester sFlt-1/PIGF ratio, and the combination of the second trimester sFlt-1 level with body mass index, were better predictors of late-onset preeclampsia than any individual marker.

Keywords: Preeclampsia, Soluble fms-like tyrosine kinase, Placental growth factor, sFlt-1, PIGF, sFlt-1/PIGF ratio, PAPP-A, BMI, Low-risk population

\footnotetext{
* Correspondence: chadh001@chamc.co.kr

${ }^{\dagger}$ Equal contributors

'Department of Obstetrics and Gynecology, CHA Gangnam Medical Center,

CHA University, Seoul, Republic of Korea

${ }^{3}$ Department of Obstetrics and Gynecology, CHA Gangnam Medical Center,

650-9 Yeoksam-dong, Gangnam-gu, Seoul, Republic of Korea

Full list of author information is available at the end of the article
} 


\section{Background}

Preeclampsia is characterized by hypertension and significant proteinuria during pregnancy. This multisystem disorder occurs in approximately $3 \%$ of pregnancies [1], and can progress to eclampsia with life-threatening seizures.

Recent evidence indicates that there are numerous phenotypes of preeclampsia, indicating that a number of pathophysiologic mechanisms related to the mother and fetus may contribute to the timing of disease onset [2]. Preeclampsia has been described as two distinct disease entities: early-onset preeclampsia, which develops before 34 weeks of gestation, and late-onset preeclampsia, which develops at or after 34 weeks of gestation [3]. Early-onset preeclampsia is strongly associated with deficient trophoblast invasion and failure of normal spiral artery remodeling. Late-onset preeclampsia may be caused by increased maternal vascular susceptibility to the normal inflammatory state of pregnancy or atherosis of a placenta that initially developed normally [4].

Preeclampsia is one of the leading causes of maternal mortality worldwide, and in developed countries it increases perinatal mortality five-fold [5]. Chronic hypertension, pregestational diabetes mellitus, multifetal pregnancy, and preeclampsia during a previous pregnancy are associated with a three- to five-fold increased risk of preeclampsia [6]. This high-risk population tends to develop severe or early preeclampsia, and accounts for about one-third of cases of early-onset preeclampsia [7]. Patients at high risk of preeclampsia usually undergo close surveillance during pregnancy, and can be referred to specialized centers for perinatal care. Women at low risk of preeclampsia also need close surveillance, and detection and prompt management of these patients can improve maternal and fetal mortality and morbidity. However, there is no reliable screening test for early identification of late-onset preeclampsia in low-risk pregnancies. Combination of the clinical, biochemical, and biophysical markers is likely to increase the predictive power of screening examinations.

It has been suggested that placental soluble fms-like tyrosine kinase 1 (sFlt-1) is a causative factor in preeclampsia. sFlt-1 acts as a potent vascular endothelial growth factor (VEGF) and placental growth factor (PIGF) antagonist by binding to these molecules and thereby reducing the free circulating levels of VEGF and PIGF. Decreased levels of circulating VEGF and PIGF appear to result in reversal of physiological vasodilation, leading to hypertension [8]. Several studies have reported that the sFlt-1/PlGF ratio can be used to identify patients at risk of preeclampsia $[9,10]$. Verlohren et al. [9] reported that a sFlt-1/PlGF ratio of $\geq 85$ (Elecsys; Roche Diagnostics GmbH, Mannheim, Germany) predicted preeclampsia. However, a lower cutoff value could yield a more sensitive diagnostic test.
In high-risk women, persistently increased uterine artery (UtA) resistance during the first half of pregnancy is associated with a high risk of early-onset preeclampsia [7]. A combination of UtA Doppler ultrasonography measurements (pulsatility index and mean arterial pressure) and biochemical markers at 11 to 13 weeks of gestation effectively identified women at high risk of hypertensive disorders in pregnancy [11]. Yu et al. [12] reported that in low-risk pregnancies, preeclampsia associated with delivery at or beyond 34 weeks of gestation was most effectively predicted by a combination of ultrasonography findings and maternal factors. However, the ability of various biophysical markers to predict preeclampsia in lowrisk pregnancies should be examined more thoroughly.

The integrated test for fetal Down's syndrome uses a combination of placental biomarkers. Among these markers, the levels of pregnancy-associated plasma protein (PAPP-A) and inhibin-A are positively associated with subsequent preeclampsia [13-16]. However, the screening efficacy of these markers for preeclampsia remains to be determined. This study evaluated the ability of the PAPP-A and inhibin-A levels, sFlt-1/PIGF ratio, and UtA Doppler ultrasonography measurements to predict preeclampsia in low-risk pregnancies, and established a cutoff value for the sFlt-1/PlGF ratio determined using the Elecsys platform.

\section{Methods}

\section{Study population}

This prospective study analyzed the risk of preeclampsia according to the results of blood sample analyses in pregnant women at low risk of preeclampsia. The study included women who received regular antenatal care at the prenatal care unit of Cha Hospital in Seoul, Korea between April 2011 and December 2011. All patients were recruited before 10 weeks of gestation, based on measurement of the fetal crown-rump length. All subjects underwent the integrated test for fetal Down's syndrome, UtA Doppler ultrasonography at 20 to 24 weeks of gestation, and calculation of the sFlt-1/PlGF ratio at 24 to 27 and 34 to 37 weeks of gestation. Women were excluded if they were aged $\geq 40$ years or had a multifetal pregnancy, chronic hypertension, prior history of preeclampsia, pregestational diabetes mellitus, gestational diabetes mellitus, delivery before 35 weeks of gestation, early-onset preeclampsia (before 35 weeks of gestation), or body mass index (BMI) $\geq 25 \mathrm{~kg} / \mathrm{m}^{2}$. All the cases of preeclampsia included in the study were diagnosed with severe preeclampsia after 35 weeks of gestation (late-onset preeclampsia), and the time of diagnosis was almost the same as the time of delivery.

Preeclampsia was defined according to the National High Blood Pressure Education Program Working Group on High Blood Pressure in Pregnancy criteria. Hypertension 
was defined as repeated systolic blood pressure measurements of $\geq 140 \mathrm{mmHg}$ (Korotkoff phase 1) and diastolic blood pressure measurements of $\geq 90 \mathrm{mmHg}$ (Korotkoff phase 5). Proteinuria was defined as $\geq 300 \mathrm{mg}$ of protein in a 24-hour urine collection sample, or repeated $\geq 1+$ proteinuria on dipstick urinalysis [17]. Preeclampsia was defined as severe if (1) the systolic blood pressure was $>160 \mathrm{mmHg}$ or the diastolic blood pressure was $>110 \mathrm{mmHg}$ on at least two occasions after 20 weeks of gestation, or (2) there was $\geq 5 \mathrm{~g}$ of protein in a 24-hour urine collection specimen or $\geq 3+$ proteinuria on dipstick urinalysis of two samples collected at least 4 hours apart. The time of onset of preeclampsia was defined as the date of diagnosis. Normal pregnancy was defined as term delivery with no documented concerns regarding hypertension or proteinuria before or after delivery.

\section{Ethics statement}

All women provided written informed consent before the collection of blood samples. The collection and use of samples for the purposes of this study were approved by the Institutional Review Board of CHA Gangnam Medical Center, CHA University.

\section{Laboratory methods}

All women underwent the integrated test for fetal Down's syndrome. Maternal serum samples were obtained at $11^{+0}$ to $13^{+6}$ and $15^{+0}$ to $20^{+6}$ weeks of gestation for measurement of the plasma PAPP-A level and the serum levels of the quadruple test markers: alphafetoprotein (MSAFP), unconjugated estriol, human chorionic gonadotropin (hCG), and inhibin-A. Markers were measured using a UniCel DxI 800 analyzer (Beckman Coulter Inc., Fullerton, CA, USA) and the values were transformed to multiples of the median (MoM) after adjusting for gestational age and maternal BMI.

To measure the plasma levels of sFlt-1 and PlGF, venous blood was collected in silicon-coated glass tubes at $24^{+0}$ to $27^{+6}$ and $34^{+0}$ to $37^{+6}$ weeks of gestation. After clotting, the samples were centrifuged and plasma was stored at $-80^{\circ} \mathrm{C}$. The sFlt- 1 and PlGF levels of each sample were measured simultaneously using the fully automated Roche Diagnostics Elecsys assay (Roche Diagnostics, Penzberg, Germany), and the sFlt-1/PlGF ratio was calculated.

\section{Uterine artery Doppler ultrasonography}

UtA Doppler transabdominal ultrasonography with color flow mapping was performed at 20 to 24 weeks of gestation. The bilateral UtA Doppler impedance indices were recorded according to the Fetal Medicine Foundation guidelines [12], including the mean pulsatility index, resistance index, systolic/diastolic ratio, and notching.

\section{Statistical analysis}

Data are expressed as the median (range) and were analyzed using non-parametric methods.

Weighted log10-linear regression was used to calculate the median values for the levels of sFlt-1, PIGF, PAPP-A, alpha-fetoprotein (MSAFP), $\beta$-hCG, unconjugated estriol, and inhibin-A, and the highest uterine artery pulsatility index, mean uterine artery pulsatility index, highest uterine artery resistance index, mean uterine artery resistance index, and nuchal translucency thickness, as a function of fetal crown-rump length and maternal weight for the 254 subjects without preeclampsia. The results for all subjects were then expressed as MoM values. Repeated analysis of variables was used to calculate the effects of gestational age on variables. Receiver operating characteristic (ROC) curve analysis was used to determine the detection rate (DR) of each marker for subsequent late-onset preeclampsia at a fixed false-positive rate (FPR). Logistic regression analysis was used to calculate the a posteriori risk of preeclampsia for each patient using the panel of available markers expressed in terms of MoM and parity.

The logistic regression equation used was $\ln (\mathrm{y})=$ $\alpha+\beta_{1} X_{1}+\beta_{2} X_{2}+\ldots \beta_{n} X_{n}$ where $\alpha$ is the constant of the model (the odds of preeclampsia without risk factors or when the risk factors assume the lowest risk value), $\beta$ is the coefficient associated with the risk factor, $X$ is the risk factor, and $y$ is the odds of preeclampsia. Therefore, odds $=\exp (\mathrm{y})$ and risk $=$ odds $/(1+$ odds $)$.

Analyses were performed using the Statistical Package for the Social Sciences version 19.0 (SPSS Inc., Chicago, IL, USA) and Power Analysis and Sample Size version 11 (NCSS LLC, Kaysville, UT, USA).

\section{Results}

A total of 262 women were enrolled in this study, of which 8 developed late-onset preeclampsia. The characteristics of subjects with and without preeclampsia and the distributions of the studied markers are shown in Table 1. There were significant differences in maternal weight before pregnancy and at delivery, BMI, infant birth weight, and parity between patients with and without preeclampsia. The BMI before pregnancy was significantly higher $(p=0.010)$, infant birth weight was significantly lower $(\mathrm{p}<0.001)$, and delivery time was significantly earlier $(\mathrm{p}<0.001)$ in patients with preeclampsia than in patients without preeclampsia. Small-for-gestational-age infants (birth weight below the $10^{\text {th }}$ percentile) were significantly more frequent in patients with preeclampsia than in patients without preeclampsia $(p=0.006)$. The MoM values of all the biochemical markers except for the MSAFP level, and of nuchal translucency thickness, were significantly different between patients with and without preeclampsia (Table 2). UtA Doppler ultrasonography measurements were not 
Table 1 Characteristics of the subjects

\begin{tabular}{llll}
\hline Variables & Preeclampsia $(\mathbf{n}=\mathbf{8})$ & Unaffected group $(\mathbf{n}=\mathbf{2 5 4})$ & $\boldsymbol{P}_{\text {-value * }}$ \\
\hline Maternal age, yrs & $33(25-37)$ & $33(20-39)$ & 0.872 \\
Nulliparous, \% & 66.1 & 50 & 0.779 \\
Prepregnancy weight $(\mathrm{kg})$ & $62(50-71)$ & $53(40-75)$ & 0.018 \\
BMl, kg/m², mean & $22.9(19.05-24.86)$ & $20.2(15.24-24.9)$ & 0.010 \\
Weight at delivery & $78(61.7-107)$ & $65(43.5-102.2)$ & 0.007 \\
Infant birth weight,gm & $2385(1760-3100)$ & $3160(2100-4000)$ & $<0.001$ \\
Small for gestational age(\%) & 37.5 & 8.7 & 0.006 \\
Gestational age at delivery & $35.5(35-40)$ & $38.5(35-41)$ & $<0.001$ \\
\hline
\end{tabular}

Data are expressed as median (minimum-maximum) or percentage.

*Mann-Whitney $U$ Test or Fisher's test.

significantly different between patients with and without preeclampsia. Figure 1 shows the results of repeated measures analysis of variance comparing the second and third trimester sFlt-1/PIGF ratios in patients with and without preeclampsia. The ratios were significantly associated with time (within-subjects effect) and the development of preeclampsia (between-subjects effect) (both $\mathrm{p}<0.001$, sphericity test).

The univariate ROC curve analyses for each of the variables evaluated are shown in Table 3 . The third trimester sFlt-1/PIGF ratio yielded the highest DR of $87.5 \%$ at a fixed FPR of $10 \%(\mathrm{p}<0.001)$, followed by the

Table 2 Distributions of the variables of interest

\begin{tabular}{|c|c|c|c|}
\hline variable & Preeclampsia $(n=8)$ & Unaffected group $(n=254)$ & $P$ value \\
\hline Median gestational age (weeks) & $11(10-13)$ & $11(10-13)$ & 0.651 \\
\hline PAPP-A, MoM & $0.63(0.34-1.67)$ & $1.00(0.20-3.85)$ & 0.046 \\
\hline NT, MoM & $1.05(0.81-1.28)$ & $1.00(0.45-1.99)$ & 0.724 \\
\hline Median gestational age & $16(15-17)$ & $16(14-18)$ & 0.784 \\
\hline MSAFP, MoM & $1.00(0.41-4.38)$ & $1.10(0.55-2,96)$ & 0.550 \\
\hline hCG,MoM & $1.66(1.16-2.68)$ & $1.00(0.20-6.53)$ & 0.001 \\
\hline uE3, MoM & $0.97(0.69-1.28)$ & $1.00(0.46-1.83)$ & 0.521 \\
\hline Inhibin A, MoM & $1.86(0.82-2.96)$ & $1.00(0.39-3.676)$ & 0.007 \\
\hline Median gestational age & $26(24-27)$ & $27(25-27)$ & 0.378 \\
\hline sFlt-1 ${ }^{1},(\mathrm{pg} / \mathrm{ml})$ & $2360(932-4435)$ & $1296(166-5710)$ & 0.001 \\
\hline $\mathrm{PIGF}^{1},(\mathrm{pg} / \mathrm{ml})$ & $219(34.25-698)$ & $491(91.37-2243)$ & 0.016 \\
\hline sFlt-1/PIGF ${ }^{1}$ & $11.9(1.7-70.9)$ & $2.6(0.3-12)$ & 0.001 \\
\hline Median gestational age & $34(34-36)$ & $36(34-37)$ & $<0.001$ \\
\hline sFlt- $1^{2},(p g / m l)$ & $6275(4307-12835)$ & $2334(71.61-50475)$ & $<0.001$ \\
\hline $\mathrm{PIGF}^{2},(\mathrm{pg} / \mathrm{ml})$ & $115.9(16.48-284.9)$ & $287.95(6.60-1749)$ & 0.001 \\
\hline sFlt-1/PIGF ${ }^{2}$ & $76.3(15.1-352.7)$ & $8(0.8-156)$ & $<0.001$ \\
\hline \multicolumn{4}{|l|}{ Uterine artery doppler } \\
\hline Mean Rl & $0.55(0.48-0.77)$ & $0.57(0.37-0.83)$ & 0.972 \\
\hline Mean PI & $0.94(0.71-1.86)$ & $0.97(0.52-2.00)$ & 0.949 \\
\hline Mean SD & $2.25(1.95-4.25)$ & $2.35(1.47-5.91)$ & 0.913 \\
\hline Highest Rl & $0.58(0.53-0.77)$ & $0.62(0.38-0.88)$ & 0.526 \\
\hline Highest PI & $1.03(0.79-1.86)$ & $1.06(0.54-2.76)$ & 0.808 \\
\hline Highest SD & $2.40(2.10-4.30)$ & $2.58(1.63-8.58)$ & 0.642 \\
\hline Notch (\%) & 0.4 & 12.5 & 0.072 \\
\hline
\end{tabular}

Data are expressed as median (minimum-maximum).

PAPP-A: pregnancy-associated plasma protein-A, NT: nuchal translucency thickness, MSAFP: alpha-fetoprotein, hCG: human chorionic gonadotropin, uE3: unconjugated estriol, sFIt- $1^{1}$ : second trimester sFIt-1, PIGF $^{1}$ : second trimester PIGF, sFIt-1/PIGF ${ }^{1}$ : second trimester sFlt-1/PIGF ratio, sFIt- ${ }^{2}$ : third trimester sFIt-1, $\mathrm{PIGF}^{2}$ : third trimester PIGF, sFlt-1/PIGF ${ }^{2}$ : thirst trimester sFlt-1/PIGF ratio. 


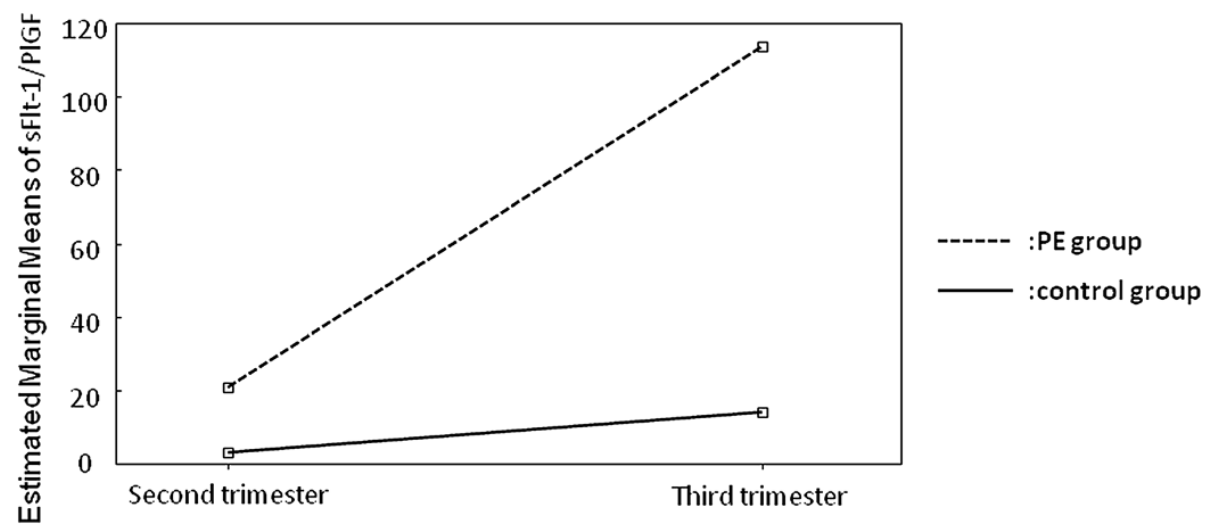

Figure 1 Slope of the sFIt-1/PIGF ratio. Results of repeated measures analysis of variance comparing the second trimester sFlt-1/PIGF ratio (sFlt-1/PIGF ${ }^{1}$ ) with the third trimester sFlt-1/PIGF ratio (sFIt-1/PIGF²). Dotted line: patients with preeclampsia, solid line: patients without preeclampsia.

second trimester sFlt-1/PlGF ratio with a DR of $75 \%$ $(p=0.001)$. Of the single markers, the sFlt-1 level yielded the best DR (75\%) at a fixed FPR of $10 \%$, followed by the PlGF level (62.5\%) and the PAPP-A level (50\%). Even though the number of preeclampsia cases was small, the two-tailed power of the data with a Type I error rate of 5\% ranged from $86 \%$ to $99 \%$ for all the ROC curves. For logistic regression analysis, an odds ratio of $>2.5$ was required to achieve sufficient power with a type I error rate of $5 \%$ at the given incidence of preeclampsia (8/262, 3\%). Figure 2 shows the univariate ROC curves for the second and third trimester sFlt-1/PlGF ratios, which had the best performance for predicting preeclampsia out of the available markers.

Multivariate ROC curves were constructed using the logistic regression analysis results (Table 4). The best performances were achieved by the combination of the PAPP-A level and the second trimester sFlt-1/PIGF ratio, and the combination of the second and third trimester sFlt-1/PlGF ratios. It is worth noting that the combination of the PAPP-A level and the second trimester sFlt-1/PlGF ratio had a DR of $87.5 \%$ (at a fixed FPR of 10\%), and the second trimester sFlt-1/PIGF ratio alone had a DR of $75 \%$ (at a fixed FPR of 10\%). The addition of BMI to the second trimester sFlt-1 level increased the DR from $75 \%$ to $87.5 \%$. The DR of the combination of the second and third trimester sFlt-1/PlGF ratios was not improved by the addition of any other marker. Interestingly, the cutoff for high risk of preeclampsia was similar for each of the five models, ranging from $2.2 \%$ to $5 \%$. Figure 3 shows multivariate ROC curves for combination models. Pattern analysis results for the cases detected by the logistic models are shown in Table 5 (right columns) and Table 6 . Three cases were detected by three of the models: the combination of the PAPP-A level and second trimester sFlt-1/PlGF ratio, the combination of the BMI and the second trimester sFlt-1 level, and the combination of the second and third trimester sFlt-1/PlGF ratios. Two cases were detected by all five models. The combination of the PAPP-A and inhibin-A levels failed to detect two of the

Table 3 Univariate ROC curve analysis for each marker and the detection rate (DR) for preeclampsia at a fixed false-positive rate (FPR) of $10 \%$ and $5 \%$

\begin{tabular}{|c|c|c|c|c|c|c|c|c|c|}
\hline Variable & Cutoff $^{1}$ & $\mathrm{DR}^{1}$ & Cutoff $^{2}$ & $\mathrm{DR}^{2}$ & Area & SE & $p$-value & $95 \% \mathrm{Cl}$ & \\
\hline $\mathrm{BMl}, \mathrm{kg} / \mathrm{m}^{2}$ & 23.6 & 37.5 & 24.3 & 25 & 0.769 & 0.086 & 0.010 & 0.600 & 0.937 \\
\hline PAPP-A, MoM & 0.58 & 50 & 0.49 & 50 & 0.707 & 0.116 & 0.046 & 0.480 & 0.935 \\
\hline Inhibin A, MoM & 1.76 & 50 & 2.18 & 50 & 0.780 & 0.095 & 0.007 & 0.593 & 0.967 \\
\hline sFlt-1 ${ }^{1},(\mathrm{pg} / \mathrm{ml})$ & 2358 & 75 & 3064 & 25 & 0.842 & 0.080 & 0.001 & 0.686 & 0.998 \\
\hline $\mathrm{sFlt}-1^{2}$ & 4680 & 75 & 6040 & 50 & 0.944 & 0.020 & $<0.001$ & 0.906 & 0.983 \\
\hline $\mathrm{PIGF}^{1},(\mathrm{pg} / \mathrm{ml})$ & 257 & 62.5 & 200 & 37.5 & 0.751 & 0.103 & 0.016 & 0.549 & 0.953 \\
\hline $\mathrm{PIGF}^{2}$ & 113.4 & 50 & 86.5 & 37.5 & 0.847 & 0.060 & 0.001 & 0.729 & 0.965 \\
\hline sFlt-1/PIGF ${ }^{1}$ & 6.05 & 75 & 7.0 & 75 & 0.851 & 0.092 & 0.001 & 0.670 & 1.000 \\
\hline sFlt-1/PIGF ${ }^{2}$ & 28.2 & 87.5 & 43.7 & 50 & 0.939 & 0.033 & $<0.001$ & 0.873 & 1.000 \\
\hline
\end{tabular}

Cutoff $^{1}$ : cutoff value at a fixed FPR of $10 \%$, DR $^{1}:$ DR at a fixed FPR of $10 \%$, Cutoff ${ }^{2}$ : cutoff value at a fixed FPR of 5\%, DR ${ }^{2}$ : DR at a fixed FPR of $5 \%$, PAPP-A: pregnancy-associated plasma protein-A, sFlt- $1^{1}$ : second trimester sFIt-1, PIGF ${ }^{1}$ : second trimester PIGF, sFlt-1/PIGF ${ }^{1}$ : second trimester sFlt-1/PIGF ratio, sFlt- $1^{2}$ : third trimester sFlt-1, $\mathrm{PIGF}^{2}$ : third trimester PIGF, sFlt-1/PIGF²: third trimester sFIt-1/PIGF ratio, SE: standard error. 


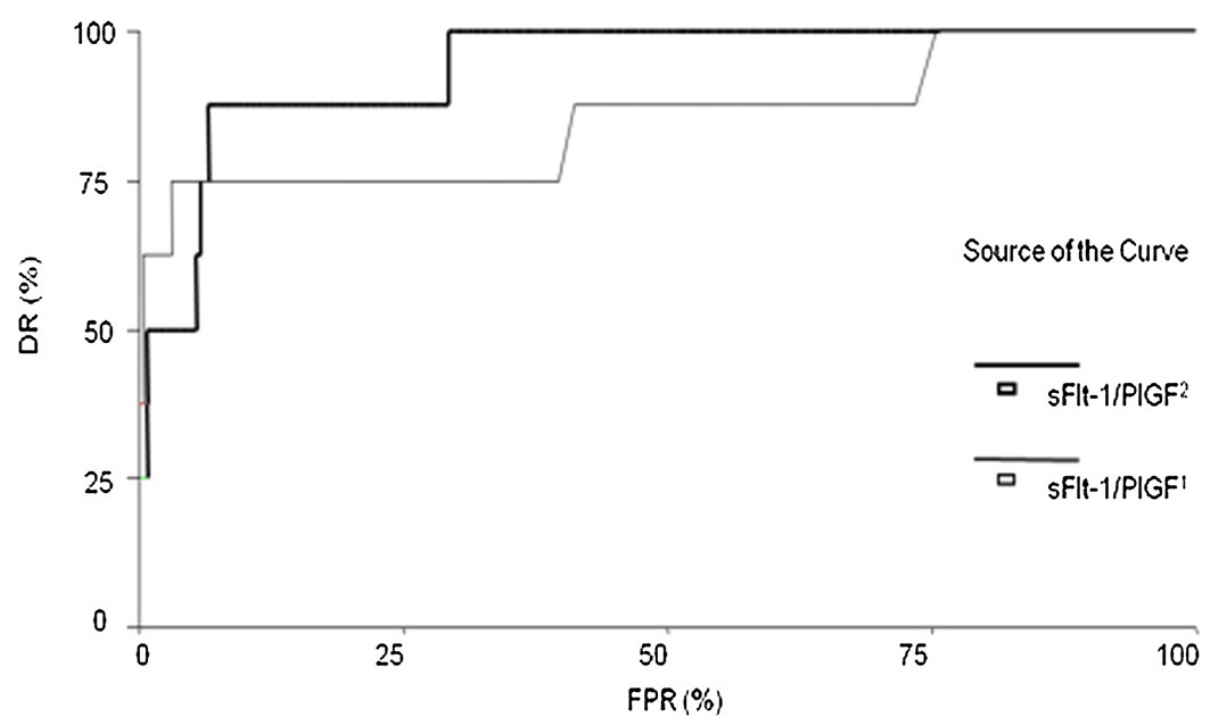

Figure 2 Univariate ROC curves for the second and third trimester sFIt-1/PIGF ratios. The second trimester sFlt-1/PIGF ratio (sFlt-1/PIGF $\left.{ }^{1}\right)$ and third trimester sFlt-1/PIGF ratio (sFlt-1/PIGF2) were the best markers for predicting preeclampsia.

remaining three cases. However, the three remaining cases were each detected by at least two models (Table 6). Parity (nulliparous versus parous) did not add any discriminatory power and was therefore excluded from the logistic models. The risk estimates for each of the eight cases of preeclampsia according to the logistic models are shown in Table 5. The different models provided quite different risk estimates for preeclampsia for the same patient. For example, Cases 4 and 218 had a risk estimation range of more than $90 \%$ (from $0.59 \%$ to $99.32 \%$ and from $5.46 \%$ to 99.99\%, respectively). This indicates that more cases need to be evaluated in future prospective studies to identify the best cutoff values for detecting patients at high risk of preeclampsia.

\section{Discussion}

This study used combinations of maternal and biochemical markers to identify women at high risk of preeclampsia in a low-risk population. We focused on late-onset preeclampsia in a low-risk population because the prevalence of late-onset preeclampsia is much higher than the prevalence of early-onset preeclampsia [18]. Early-onset preeclampsia is usually associated with placental dysfunction, reduction in placental volume, intrauterine growth restriction, abnormal uterine and umbilical artery Doppler ultrasonography findings, low birth weight, multi-organ dysfunction, perinatal death, and adverse maternal and neonatal outcomes $[19,20]$. Late-onset preeclampsia is more often associated with a normal placenta, larger placental volume, normal fetal growth, normal uterine and umbilical artery Doppler ultrasonography findings, normal birth weight, and more favorable maternal and neonatal outcomes [19,21,22]. Considering the heterogeneous pathophysiology, no single screening test is sufficient for predicting preeclampsia.

Our results indicate that combination of the Elecsys sFlt-1/PlGF ratio with the pre-pregnancy BMI and biochemical markers during pregnancy may improve the sensitivity of predicting preeclampsia in a low-risk population. The DRs for individual markers ranged from

Table 4 ROC curve analyses for combinations of markers and the DR for preeclampsia at a fixed FPR of $10 \%$ and $5 \%$ using a logistic regression model

\begin{tabular}{|c|c|c|c|c|c|c|c|c|c|}
\hline Variable & Cutoff $^{1}$ & $\mathrm{DR}^{1}$ & Cutoff $^{2}$ & $\mathrm{DR}^{2}$ & Area & SE & p-value & $95 \% \mathrm{Cl}$ & Variable \\
\hline PAPP-A + Inhibin A & $5.01 \%$ & 50 & $9.19 \%$ & 37.5 & 0.812 & 0.093 & 0.003 & 0.629 & 0.995 \\
\hline PAPP-A + sFlt-1/PIGF ${ }^{1}$ & $2.85 \%$ & 87.5 & $4.72 \%$ & 87.5 & 0.969 & 0.015 & $<0.001$ & 0.940 & 0.999 \\
\hline PAPP-A + sFlt-1/PIGF ${ }^{2}$ & $2.62 \%$ & 62.5 & $4.07 \%$ & 50 & 0.917 & 0.036 & $<0.001$ & 0.847 & 0.988 \\
\hline $\mathrm{BMI}+\mathrm{sFlt}-1^{1}$ & $4.6 \%$ & 87.5 & $8.9 \%$ & 75 & 0.937 & 0.039 & $<0.001$ & 0.861 & 1.000 \\
\hline sFlt-1/PIGF ${ }^{1}+$ sFlt-1/PIGF ${ }^{2}$ & $2.2 \%$ & 87.5 & $3.4 \%$ & 87.5 & 0,946 & 0.043 & $<0.001$ & 0.862 & 1.000 \\
\hline
\end{tabular}

The exploratory variable in each case is the risk determined by the logistic equation.

Cutoff $^{1}$ : cutoff value at a fixed FPR of $10 \%, D^{1}:$ DR at a fixed FPR of $10 \%$, Cutoff ${ }^{2}$ : cutoff value at a fixed FPR of $5 \%$, DR ${ }^{2}$ : DR at a fixed FPR of $5 \%$, PAPP-A: pregnancy-associated plasma protein-A, sFIt-1 ${ }^{1}$ : second trimester sFIt-1, sFIt-1/PIGF ${ }^{1}$ : second trimester sFlt-1/PIGF ratio, sFIt-1/PIGF ${ }^{2}$ : third trimester sFlt-1/PIGF ratio, SE: standard error. 


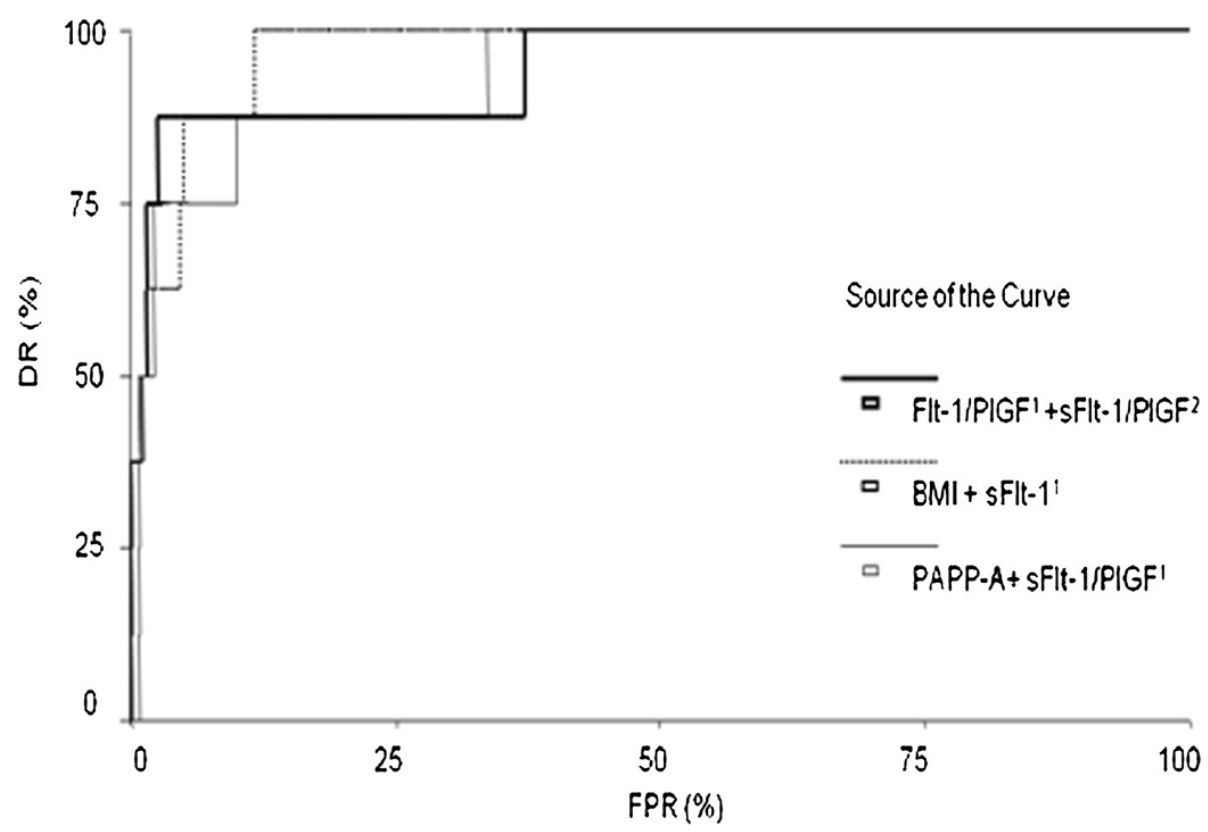

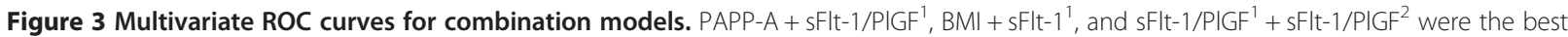
combinations for predicting preeclampsia. PAPP-A: pregnancy-associated plasma protein-A, sFlt-1 ${ }^{1}$ : second trimester sFlt-1, sFlt-1/PIGF ${ }^{1}$ : second trimester sFlt-1/PIGF ratio, sFlt-1/PIGF²: third trimester sFlt-1/PIGF ratio.

$37.5 \%$ to $87.5 \%$ for a fixed FPR of $10 \%$, and from $25 \%$ to $75 \%$ for a fixed FPR of $5 \%$. After combining markers, the estimated DR ranged from $50 \%$ to $87.5 \%$ for a fixed FPR of $10 \%$, and from $37.5 \%$ to $87.5 \%$ for a fixed FPR of $5 \%$. Combining markers had a moderate benefit at a fixed FPR of $5 \%$. The second and third trimester sFlt-1 levels had the highest DRs among the single markers, but the sFlt-1/PlGF ratio was a stronger predictor of preeclampsia than either marker alone. The third trimester sFlt-1/ PlGF ratio had the highest DR of the individual variables evaluated, but was not sufficient as a single screening method for early detection of preeclampsia.

ROC curve analysis showed that BMI was the strongest clinical predictor of preeclampsia with a DR of $37.5 \%$ at a fixed FPR of $10 \%$ and a DR of $25 \%$ at a fixed FPR of $5 \%$. A previous study reported that increased early pregnancy insulin resistance was independently associated with subsequent preeclampsia [23]. We found that BMI predicted preeclampsia $(\mathrm{p}=0.010)$ at a cutoff value of $23.64 \mathrm{~kg} / \mathrm{m}^{2}$ at a fixed FPR of $10 \%$ and $24.26 \mathrm{~kg} / \mathrm{m}^{2}$ at a fixed FPR of $5 \%$, even in a low-risk population with $\mathrm{BMI}<25 \mathrm{~kg} / \mathrm{m}^{2}$. This result is consistent with previous reports that obese women are at increased risk of developing preeclampsia [24,25].

Duckitt and Harrington [26] performed a systematic review of 52 studies and found that nulliparity was a risk factor for preeclampsia (relative risk: 2.91). A prospective cohort study also found that nulliparous women had

Table 5 Estimated risk (\%) for each case of preeclampsia for each model at a fixed FPR of $10 \%$

\begin{tabular}{|c|c|c|c|c|c|c|c|c|c|c|}
\hline Cases ID & PAPP-A + Inhibin A & PAPP-A + sFIt-1/PIGF ${ }^{1}$ & PAPP-A + sFIt-1/PIGF ${ }^{2}$ & $\mathrm{BMI}+\mathrm{sFlt}-1^{1}$ & sFIt-1/PIGF ${ }^{1}+$ sFlt-1/PIGF ${ }^{2}$ & & \multicolumn{4}{|c|}{ Pattern* } \\
\hline 38 & 4.43 & 2.59 & 55.44 & 0.79 & 7.25 & 0 & 0 & 1 & 0 & 1 \\
\hline 51 & 18.64 & 4.76 & 2.62 & 5.30 & 0.64 & 1 & 1 & 1 & 1 & 0 \\
\hline 147 & 2.86 & 5.40 & 1.62 & 51.4 & 19.85 & 0 & 1 & 0 & 1 & 1 \\
\hline 137 & 36.53 & 33.60 & 38.30 & 19.1 & 31.95 & 1 & 1 & 1 & 1 & 1 \\
\hline 144 & 2.96 & 36.02 & 3.11 & 42.9 & 20.90 & 0 & 1 & 1 & 1 & 1 \\
\hline 4 & 0.59 & 99.32 & 1.88 & 40.3 & 97.83 & 0 & 1 & 0 & 1 & 1 \\
\hline 218 & 5.46 & 99.99 & 80.35 & 20.8 & 99.99 & 1 & 1 & 1 & 1 & 1 \\
\hline 153 & 26.96 & 100 & 99.92 & 30.6 & 100 & 1 & 1 & 1 & 1 & 1 \\
\hline
\end{tabular}

*0 = missed case, 1 = detected case.

PAPP-A: pregnancy-associated plasma protein-A, sFlt-1 ${ }^{1}$ : second trimester sFlt-1, $\mathrm{PIGF}^{1}$ : second trimester PIGF, sFlt-1/PIGF ${ }^{1}$ : second trimester sFlt-1/PIGF ratio, sFIt-12: third trimester sFlt-1, PIGF²: third trimester PIGF, sFlt-1/PIGF²: third trimester sFlt-1/PIGF ratio. 
Table 6 Patterns analysis for detection of preeclampsia for each model at a fixed FPR of $10 \%$

\begin{tabular}{|c|c|c|c|c|c|}
\hline \multirow[t]{2}{*}{ Number of cases } & \multicolumn{5}{|c|}{ Detection model of preeclampsia* } \\
\hline & PAPP-A + Inhibin A & PAPP-A + sFlt-1/PIGF ${ }^{1}$ & PAPP-A + sFlt-1/PIGF ${ }^{2}$ & $\mathrm{BMI}+\mathrm{sFlt}-1^{1}$ & sFIt-1/PIGF ${ }^{1}+$ sFlt-1/PIGF \\
\hline 1 & 0 & 0 & 1 & 0 & 1 \\
\hline 2 & 0 & 1 & 0 & 1 & 1 \\
\hline 1 & 0 & 1 & 1 & 1 & 1 \\
\hline 1 & 1 & 1 & 1 & 1 & 0 \\
\hline 3 & 1 & 1 & 1 & 1 & 1 \\
\hline
\end{tabular}

${ }^{*} 0=$ missed case, 1 = detected case

PAPP-A: pregnancy-associated plasma protein-A, sFlt-1 ${ }^{1}$ : second trimester sFlt-1, $\mathrm{PIGF}^{1}$ : second trimester PIGF, sFlt-1/PIGF ${ }^{1}$ : second trimester sFIt-1/PIGF ratio, sFlt- $1^{2}$ : third trimester sFlt-1, $\mathrm{PIGF}^{2}$ : third trimester PIGF, sFlt-1/PIGF ${ }^{2}$ : third trimester sFIt-1/PIGF ratio.

higher blood pressure levels throughout pregnancy and higher risks of uterine artery notching and gestational hypertensive disorders than other women [27]. However, no association between preeclampsia and parity was observed in this study $(p=0.779)$. This difference may result from differences in ethnicity and marital status between the subjects in the different studies, or the relatively small number of cases in this study.

The integrated test for fetal Down's syndrome is based on the levels of several first and second trimester fetoplacental markers. PAPP-A is a large, highly glycosylated protein that is produced by developing trophoblast cells. PAPP-A has been shown to be a syncytiotrophoblastderived insulin-like growth factor binding protein protease [28]. A multicenter study of 8,839 women demonstrated a significant relationship between a PAPP-A level at or below the $5^{\text {th }}$ percentile and intrauterine growth restriction, preterm delivery, preeclampsia, and stillbirth [13]. Inhibin-A is a glycoprotein hormone that is a member of the transforming growth factor- $\beta$ family. The placenta is the primary source of this circulating protein during pregnancy, and its concentration increases in the third trimester of uncomplicated pregnancies [29]. Although an increased serum inhibin-A level is significantly associated with subsequent preeclampsia, the inhibin-A level has poor sensitivity for predicting preeclampsia [14,15]. Nevertheless, we found that the inhibin-A level was a more sensitive marker for predicting subsequent preeclampsia than the hCG level in the second trimester. Addition of the hCG level to the inhibin-A level did not improve the screening efficacy for preeclampsia in a previous study [16]. Significantly lower PAPP-A levels and higher inhibin-A and hCG levels were observed in women who developed preeclampsia than in those who did not, but the levels of unconjugated estriol and MSAFP had no predictive power. At a fixed FPR of $10 \%$ and $5 \%$, the PAPP-A and inhibin-A levels both had DRs of $50 \%$. In our hospitals, the integrated test for fetal Down's syndrome is routinely offered to all women. This test includes the PAPP-A level at 11 to 13 weeks of gestation and the inhibin-A level at 15-20 weeks of gestation. These biochemical markers for Down's syndrome could potentially be used to improve the prediction of preeclampsia.

Recent studies have shown that UtA Doppler waveform analysis can identify women at risk of adverse pregnancy outcomes in the second trimester [30-32]. As described above, abnormal placentation is considered to play a central role in the pathogenesis of preeclampsia. Shallow cytotrophoblast interstitial invasion and failure of endovascular invasion result in increased vascular resistance and decreased placental perfusion [32]. However, this mechanism is more relevant in early-onset preeclampsia than in late-onset preeclampsia. A recent study proposed that 22 to 25 weeks of gestation is the best time for predicting preeclampsia by uterine artery Doppler velocimetry data [33]. Costa et al. [34] reported that second trimester UtA Doppler ultrasonography measurements had high sensitivity for predicting preeclampsia in a low-risk population, but only a $29 \%$ positive predictive value. A low positive predictive value for late-onset disease can complicate screening in a low-risk population. In our study, we found no significant association between UtA Doppler ultrasonography measurements and preeclampsia. Our small sample size and the fact that all cases of preeclampsia were late-onset disease might have contributed to our failure to detect a significant association between UtA Doppler ultrasonography measurements and preeclampsia.

The Elecsys system is an immunoassay system that measures plasma sFlt-1 and PlGF levels. Although it was developed for diagnostic testing of patients with preeclampsia, a carefully established cutoff value could potentially enable detection of subclinical preeclampsia. A recent study demonstrated patients with preeclampsia or the syndrome of hemolysis, elevated liver enzymes, and low platelet count with a high sFlt-1/PlGF ratio were at significantly increased risk of imminent delivery, and the authors proposed that the sFlt-1/PlGF ratio be used as a prognostic marker [10]. In our study, blood samples for the Elecsys assay were collected at $24^{+0}$ to $27^{+6}$ and $34^{+0}$ 
to $37^{+6}$ weeks of gestation. The sFlt- 1 level yielded a DR of $25 \%$ at a fixed FPR of $10 \%$, and a DR of $75 \%$ at a fixed FPR of $5 \%$. The PIGF level yielded a lower DR than the sFlt-1 level at a fixed FPR of 5\%, in the first trimester but not in the second trimester. The second trimester sFlt-1/ PIGF ratio yielded a DR of $75 \%$ at a fixed FPR of $10 \%$ (cutoff value: 6.05 ) and 5\% (cutoff value: 7.0 ). The third trimester sFlt-1/PlGF ratio yielded a DR of $87.5 \%$ at a fixed FPR of $10 \%$ (cutoff value: 28.15 ) and a DR of $50 \%$ at a fixed FPR of $5 \%$ (cutoff value: 43.70). The Elecsys instructions recommend using an sFlt-1/PlGF ratio of $>85$ (independent of gestational age) for diagnosing preeclampsia. The results of this study show that the sFlt-1/PlGF ratio is a useful predictor of late-onset preeclampsia. Ohkuchi et al. [35] reported that a cutoff value of 45 for the Elecsys sFlt-1/PlGF ratio gave 100\% sensitivity and 95\% specificity for detecting preeclampsia in a Japanese population. Soto et al. [36] reported that women with late-onset preeclampsia had significantly lower PIGF and soluble VEGF receptor-2 levels, lower PlGF/soluble endoglin and PlGF/soluble VEGF receptor-1 ratios, and higher soluble VEGF receptor-1 and soluble endoglin levels than normal pregnant women. Evidence of an imbalance between angiogenic and anti-angiogenic factors in preeclampsia has consistently been observed in women of Hispanic, Caucasian, Asian, African, and AfricanAmerican origin, and therefore appears to be a consistent finding of this disorder [36].

In this study, small-for-gestational-age infants were usually associated with preeclampsia. In patients without preeclampsia, the median sFlt-1/PlGF ratio at 34 to 37 weeks of gestation was low but had a very wide range (median: 8; range: 0.8-156). Four patients had an sFlt-1/ PlGF ratio of $>85$. Among these patients, the mean UtA pulsatility index was above the $95^{\text {th }}$ percentile for gestational age [37] in only one case with a small-for-gestational-age infant.

In this study, the combination of the second and third trimester sFlt-1/PlGF ratios and the combination of the PAPP-A level and the second trimester sFlt-1/ PIGF ratio yielded the best DRs. The sFlt-1/PIGF ratio measured shortly before delivery at or beyond 34 weeks of gestation has little value as a screening test, because delivery is an effective treatment for lateonset preeclampsia. However, if the second trimester sFlt-1/PlGF ratio is equivocal and the patient does not have preeclampsia at 34 weeks of gestation, serial measurements may help clinicians to detect and manage emerging late-onset preeclampsia. The combination of the PAPP-A level and the second trimester sFlt-1/PlGF ratio is therefore the best marker for early detection of lateonset preeclampsia.

Akolekar et al. [38] developed an effective firsttrimester screening model for preeclampsia using the
UtA pulsatility index, mean arterial pressure, and PAPP$\mathrm{A}$ and PlGF MoM values. Their algorithms detected 95.3\% of cases of early-onset preeclampsia and $45.6 \%$ of cases of late-onset preeclampsia at a fixed FPR of $10.9 \%$. Saxena et al. [39] reported that the mean third trimester sFlt-1 level was significantly higher $(p=0.002)$, and the mean first trimester sFlt-1 level was significantly lower $(\mathrm{p}=0.03)$, in women who developed preeclampsia than in women with normal pregnancies. The serial measurements of the sFlt-1/PlGF ratio used in this study enabled a much higher rate of detection of late-onset preeclampsia than the $45.6 \%$ described above.

A weak point of our study is the different risk estimation results obtained when different combinations of markers were used in the same subject. This is probably due to the low number of patients with preeclampsia, and the fact that preeclampsia results from various pathophysiologic mechanisms that may alter the markers in different ways. However, three of the eight cases were detected by all the models used, and all the cases were detected by at least two of the models. The strong points of the study include its prospective design and the evaluation of several markers for predicting preeclampsia. The combinations used in this study appear to have higher predictive accuracy than the combination of firsttrimester maternal risk factors and mean arterial pressure used in the large prospective study by Poon et al. [40], which reported a 52\% detection rate for preeclampsia at a $10 \%$ false-positive rate. A larger number of patients may have resulted in different accuracy rates for the combinations evaluated in our study. However, there was a steep increase in the sFlt-1/PlGF ratio in patients with preeclampsia (Figure 1), which is consistent with previously reported results [41], and we expect that the main conclusions would not change with a larger study population. Although the slope of the sFlt-1/PlGF ratio was not used in our algorithms, this value may be useful for enhancing predictive accuracy in a future larger scale study.

\section{Conclusions}

The Elecsys sFlt-1/PlGF ratio allows identification of low-risk pregnancies at high risk of developing preeclampsia. A cutoff value of 6.05 in the second trimester and 28.15 in the third trimester had the best ability to predict preeclampsia at a fixed FPR of $10 \%$. The PAPP-A level and BMI can be used in combination with the sFlt-1/PIGF ratio to increase the rate of detection of preeclampsia. Further studies are required to assess the usefulness of our combined screening test in low-risk populations.

\section{Competing interests}

All authors declare that they have no competing interests. 


\section{Authors' contributions}

DHC and KJL conceived of the study. HJP, DHC, KJL and SHK participated in the study design. AF and MZ performed the statistical analyses. HJP, AF and $M Z$ interpreted the data. HJP drafted and revised the manuscript. HJP, DHC KLL, SHK, SSS, JYK and YKC made substantial contributions to the acquisition of data. DHC, KLL, YWJ and AF critically reviewed the manuscript. All authors read and approved the final manuscript.

\section{Acknowledgements}

This study was supported by a restricted grant from Roche. The funder had no role in the study design, data collection and analysis, decision to publish, or preparation of the manuscript.

\section{Author details}

'Department of Obstetrics and Gynecology, CHA Gangnam Medical Center, CHA University, Seoul, Republic of Korea. ${ }^{2}$ Department of Medicine and Surgery (DIMEC), Division of Prenatal Medicine, University of Bologna, Bologna, Italy. ${ }^{3}$ Department of Obstetrics and Gynecology, CHA Gangnam Medical Center, 650-9 Yeoksam-dong, Gangnam-gu, Seoul, Republic of Korea.

Received: 28 September 2013 Accepted: 14 January 2014

Published: 20 January 2014

\section{References}

1. World Health Organization, et al: World Health Report 2005: make every mother and child count. Geneva: chapter three- great expectations: making pregnancy safer; 2005:41-58.

2. Phillips JK, Janowiak M, Badger GJ, Bernstein IM: Evidence for distinct preterm and term phenotypes of preeclampsia. J Matern Fetal Neonatal Med 2010, 23(7):622-626

3. Raymond D, Peterson E: A critical review of early-onset and late-onset preeclampsia. Obstet Gynecol Surv 2011, 66(8):497-506.

4. Costa Fda S, Murthi P, Keogh R, Woodrow N: Early screening for preeclampsia. Rev Bras Ginecol Obstet 2011, 33(11):367-375.

5. Roberts JM, Pearson GD, Cutler JA, Lindheimer MD: Summary of the NHLBI Working Group on Research on Hypertension during Pregnancy. Hypertens Pregnancy 2003, 22(2):109-127.

6. Caritis S, Sibai B, Hauth J, Lindheimer MD, Klebanoff M, Thom E, VanDorsten P, Landon M, Paul R, Miodovnik M, et al: Low-dose aspirin to prevent preeclampsia in women at high risk. National Institute of Child Health and Human Development Network of Maternal-Fetal Medicine Units. N Engl I Med 1998, 338(11):701-705.

7. Herraiz I, Escribano D, Gomez-Arriaga Pl, Hernindez-Garcia JM, Herraiz MA, Galindo A: Predictive value of sequential models of uterine artery Doppler in pregnancies at high risk for pre-eclampsia. Ultrasound Obstet Gynecol 2012, 40(1):68-74.

8. Maynard SE, Min JY, Merchan J, Lim KH, Li J, Mondal S, Libermann TA Morgan JP, Sellke FW, Stillman IE, et al: Excess placental soluble fms-like tyrosine kinase 1 (sFlt1) may contribute to endothelial dysfunction, hypertension, and proteinuria in preeclampsia. J Clin Invest 2003, 111(5):649-658.

9. Verlohren S, Galindo A, Schlembach D, Zeisler H, Herraiz I, Moertl MG, Pape J, Dudenhausen JW, Denk B, Stepan H: An automated method for the determination of the sFIt-1/PIGF ratio in the assessment of preeclampsia. Am J Obstet Gynecol 2010, 202(2):161.e1-161.e11.

10. Verlohren S, Herraiz I, Lapaire O, Schlembach D, Moertl M, Zeisler H, Calda P, Holzgreve W, Galindo A, Engels T, et al: The sFIt-1/PIGF ratio in different types of hypertensive pregnancy disorders and its prognostic potential in preeclamptic patients. Am J Obstet Gynecol 2012, 206(1):58. e51-58.

11. Poon LC, Akolekar R, Lachmann R, Beta J, Nicolaides KH: Hypertensive disorders in pregnancy: screening by biophysical and biochemical markers at 11-13 weeks. Ultrasound Obstet Gynecol 2010, 35(6):662-670

12. Yu CK, Smith GC, Papageorghiou AT, Cacho AM, Nicolaides KH: An integrated model for the prediction of preeclampsia using maternal factors and uterine artery Doppler velocimetry in unselected low-risk women. Am J Obstet Gynecol 2005, 193(2):429-436.

13. Smith GC, Stenhouse EJ, Crossley JA, Aitken DA, Cameron AD, Connor JM: Early pregnancy levels of pregnancy-associated plasma protein a and the risk of intrauterine growth restriction, premature birth, preeclampsia, and stillbirth. J Clin Endocrinol Metab 2002, 87(4):1762-1767.
14. Muttukrishna S, North RA, Morris J, Schellenberg JC, Taylor RS, Asselin J, Ledger W, Groome N, Redman CW: Serum inhibin A and activin A are elevated prior to the onset of pre-eclampsia. Hum Reprod 2000, 15(7):1640-1645.

15. Sibai BM, Koch MA, Freire S, Silva JL P e, Rudge MV, Martins-Costa S, Bartz J, de Barros Santos C, Cecatti JG, Costa R, et al: Serum inhibin A and angiogenic factor levels in pregnancies with previous preeclampsia and/or chronic hypertension: are they useful markers for prediction of subsequent preeclampsia? Am J Obstet Gynecol 2008, 199(3):268. e261-269.

16. Aquilina J, Maplethorpe R, Ellis P, Harrington K: Correlation between second trimester maternal serum inhibin- $A$ and human chorionic gonadotrophin for the prediction of pre-eclampsia. Placenta 2000 21(5-6):487-492

17. Report of the National High Blood Pressure Education Program Working Group on High Blood Pressure in Pregnancy. Am J Obstet Gynecol 2000, 183(1):S1-S22

18. Sibai BM: Diagnosis and management of gestational hypertension and preeclampsia. Obstet Gynecol 2003, 102(1):181-192.

19. Obed S, Patience A: Birth weight and ponderal index in pre-eclampsia: a comparative study. Ghana Med J 2006, 40(1):8-13.

20. Ihle BU, Long P, Oats J: Early onset pre-eclampsia: recognition of underlying renal disease. BMJ 1987, 294(6564):79-81.

21. Crispi F, Llurba E, Dominguez C, Martin-Gallan P, Cabero L, Gratacos E: Predictive value of angiogenic factors and uterine artery Doppler for early- versus late-onset pre-eclampsia and intrauterine growth restriction. Ultrasound Obstet Gynecol 2008, 31(3):303-309.

22. Dissanayake VH, Samarasinghe HD, Morgan L, Jayasekara RW, Seneviratne HR, Broughton Pipkin F: Morbidity and mortality associated with pre-eclampsia at two tertiary care hospitals in Sri Lanka. J Obstet Gynaecol Res 2007, 33(1):56-62.

23. Wolf M, Sandler L, Munoz K, Hsu K, Ecker JL, Thadhani R: First trimester insulin resistance and subsequent preeclampsia: a prospective study. J Clin Endocrinol Metab 2002, 87(4):1563-1568.

24. Marshall NE, Guild C, Cheng YW, Caughey AB, Halloran DR: Maternal superobesity and perinatal outcomes. Am J Obstet Gynecol 2012, 206(5):417. e411-416.

25. Lee CJ, Hsieh TT, Chiu TH, Chen KC, Lo LM, Hung TH: Risk factors for pre-eclampsia in an Asian population. Int I Gynaecol Obstet 2000 70(3):327-333

26. Duckitt K, Harrington D: Risk factors for pre-eclampsia at antenatal booking: systematic review of controlled studies. BMJ 2005, 330(7491):565.

27. Rurangirwa AA, Gaillard R, Steegers EA, Hofman A, Jaddoe W: Hemodynamic adaptations in different trimesters among nulliparous and multiparous pregnant women; the Generation R study. Am J Hypertens 2012, 25(8):892-899.

28. Lawrence JB, Oxvig C, Overgaard MT, Sottrup-Jensen L, Gleich GJ, Hays LG Yates JR 3rd, Conover CA: The insulin-like growth factor (IGF)-dependent IGF binding protein-4 protease secreted by human fibroblasts is pregnancy-associated plasma protein-A. Proc Natl Acad Sci USA 1999, 96(6):3149-3153.

29. Petraglia F, Vaughan J, Vale W: Inhibin and activin modulate the release of gonadotropin-releasing hormone, human chorionic gonadotropin, and progesterone from cultured human placental cells. Proc Natl Acad Sci USA 1989, 86(13):5114-5117.

30. Papageorghiou AT, Yu CK, Bindra R, Pandis G, Nicolaides KH: Multicenter screening for pre-eclampsia and fetal growth restriction by transvaginal uterine artery Doppler at 23 weeks of gestation. Ultrasound Obstet Gynecol 2001, 18(5):441-449.

31. Cnossen JS, Morris RK, ter Riet G, Mol BW, van der Post JA, Coomarasamy A, Zwinderman AH, Robson SC, Bindels PJ, Kleijnen J, et al: Use of uterine artery Doppler ultrasonography to predict pre-eclampsia and intrauterine growth restriction: a systematic review and bivariable meta-analysis. CMAJ 2008, 178(6):701-711.

32. Roberts JM, Cooper DW: Pathogenesis and genetics of pre-eclampsia. Lancet 2001, 357(9249):53-56.

33. Parra M, Rodrigo R, Barja P, Bosco C, Fernandez V, Munoz H, Soto-Chacon E: Screening test for preeclampsia through assessment of uteroplacental blood flow and biochemical markers of oxidative stress and endothelial dysfunction. Am J Obstet Gynecol 2005, 193(4):1486-1491. 
34. da Silva CF, da Cunha SP, Berezowsky AT: Dopplervelocimetria das artérias uterinas na predição de complicações em gestações de baixo risco. Rev Bras Ginecol Obstet 2005, 27(6):323-330.

35. Ohkuchi A, Hirashima C, Suzuki H, Takahashi K, Yoshida M, Matsubara S, Suzuki M: Evaluation of a new and automated electrochemiluminescence immunoassay for plasma sFIt-1 and PIGF levels in women with preeclampsia. Hypertens Res 2010, 33(5):422-427.

36. Soto E, Romero R, Kusanovic JP, Ogge G, Hussein Y, Yeo L, Hassan SS, Kim CJ, Chaiworapongsa T: Late-onset preeclampsia is associated with an imbalance of angiogenic and anti-angiogenic factors in patients with and without placental lesions consistent with maternal underperfusion. J Matern Fetal Neonatal Med 2012, 25(5):498-507.

37. Arduini D, Rizzo G: Normal values of Pulsatility Index from fetal vessels: a cross-sectional study on 1556 healthy fetuses. J Perinat Med 1990, 18(3):165-172.

38. Poon LC, Syngelaki A, Akolekar R, Lai J, Nicolaides KH: Combined screening for preeclampsia and small for gestational age at 11-13 weeks. Fetal Diagn Ther 2013, 33(1):16-27.

39. Saxena AR, Seely EW, Rich-Edwards JW, Wilkins-Haug LE, Karumanchi SA, McElrath TF: First trimester PAPP-A levels correlate with sFIt-1 levels longitudinally in pregnant women with and without preeclampsia. BMC Pregnancy Childbirth 2013, 13:85.

40. Poon LC, Kametas NA, Valencia C, Chelemen T, Nicolaides KH: Hypertensive disorders in pregnancy: screening by systolic diastolic and mean arterial pressure at 11-13 weeks. Hypertens Pregnancy 2011, 30(1):93-107.

41. Schoofs K, Grittner U, Engels T, Pape J, Denk B, Henrich W, Verlohren S: The importance of repeated measurements of the sFIt-1/PIGF ratio for the prediction of preeclampsia and intrauterine growth restriction. J Perinat Med 2014, 42(1):61-68.

doi:10.1186/1471-2393-14-35

Cite this article as: Park et al: Screening models using multiple markers for early detection of late-onset preeclampsia in low-risk pregnancy. BMC Pregnancy and Childbirth 2014 14:35.

\section{Submit your next manuscript to BioMed Central and take full advantage of:}

- Convenient online submission

- Thorough peer review

- No space constraints or color figure charges

- Immediate publication on acceptance

- Inclusion in PubMed, CAS, Scopus and Google Scholar

- Research which is freely available for redistribution 